\title{
Automatic Gate using Face Recognition Technique using HAAR Cascade Algorithm
}

\author{
N. Dileep kumar, S. Shanthi
}

\begin{abstract}
Now a day, in every single person households it is important to check regularly regarding their safety. Especially for elderly people it is mandatory, because they have become a target for certain burglars which leads to higher accidents/robberies in almost all the areas. To decrease the risk of such unwanted happenings in living space for single-person households, the hybrid security system should be adopted. The automatic personal identification has become the popular instead of using passwords or pattern in this days. This paper addresses the development of a face recognition technique for the above mentioned purpose.
\end{abstract}

Keywords: - Security system, Face Recognition, HAAR cascade algorithm, Raspberry Pi.

\section{INTRODUCTION}

Security systems have suggested various preferred approaches like biometric and password to enhance security. The technology is developing day by day and growing with the usage of different equipment's. The trend has been moved from fingerprint to face recognition. So face recognition technique has been utilized in this system for unlocking the door. Facial recognition technology can be used in various industries and corporate sectors. This is about door unlocking system that mainly uses facial recognition technology. The camera is used to capture the face images and the images are sent to the database. If the image matches with the administration image then door will be unlocked and an acknowledgement is saved in the database system. If face is not matched, then a warning message will be sent to the admin device, as someone is trying to unlock the door.

\section{LITERATURE SURVEY}

Many works have been carried in this area of security system. Some of the relevant works have been reviewed here in this section. In the door access system done by Vamsi krishna thulluri et al., [1] face recognition the images are stored in a data base. The data processing has been done by Raspberry Pi. In this, the has been used. This would give more accurate results compared to other types of s such as Fisher Face, Eygen Faces s. This will take many numbers of images as you wish in different angles and check those all images at the time of face no recognition.

Revised Manuscript Received on February 05, 2020.

* Correspondence Author

N. Dileep kumar, Electronics and Communication Engineering, Saveetha School of Engineering, SIMATS Chennai, Tamilnadu, India. Email: Dk8019436260@gmail.com.

S. Shanthi, Electronics and Communication Engineering, Saveetha School of Engineering, SIMATS Chennai, Tamilnadu, India. E-mail: shanthis.sse@saveetha.com.

(C) The Authors. Published by Blue Eyes Intelligence Engineering and Sciences Publication (BEIESP). This is an open access article under the CC BY-NC-ND license (http://creativecommons.org/licenses/by-nc-nd/4.0/)
But in this work, 20 images of a person have been taken with different angles and it stored in the date base. In the author used VNC viewer to run raspberry operating system for detecting images from the data base. At first, he has saved images by using data sets and after that, he will train that faces to then it stores into the data base. At first, it converts color images to gray scale images and then it converts into pixels for detecting this will divides the image into various pieces then it stores the values of each pixel. If pixels are less then it will be represented as 0 and pixels which are high will be 1 then it will be arranged in $3 \times 3$ matrix format for recognizing the new images on screen compared to data base stored image [vamsi krishna thulluri2019][1].

The author Harish Balkandhe et al., proposed by using face recognition and along with the e-Mail alert machine has been presented. This device has been used with home door access lock control primarily based on face reputation method by verifying enrolled facial images. The camera will scan the face if the face matches it will unlock the door. If the face doesn't match the scanned image will be sent to the admin mail [Harish Balkandh-2018][2].

In the system Hla Myo Tun et al., explains that Face reputation that does not require to be touched with any hardware. Face is detected automatically via the face detection method and the complete face reputation is completed with out touching with any hardware. By this face detection, it may identify best the facial part of an photo irrespective of the historical past of the photo. In this system, the writer used Viola-Jones face detection method . Viola-Jones re-scale the detector instead of the input photograph the detector many times thru the picture on every occasion with the exclusive size. Viola-Jones has devised a scale invariant detector that requires the equal range of calculations whatever may be the size. This detector is built using Haar wavelets. In this Face recognition typically includes function extraction, feature discount and recognition [Hla Myo Tun-2018][3].

The concept explained by the Modela gopi et al., that in present days, safety is required because of the pinnacle of the line resources individuals obtain. Because of the improvement in splitting security frameworks there's a high need in growing pinnacle of the road protection frameworks which are hard to hack. So whilst we plan a safety framework which utilizes confront as a get to key it is tough to break these. This will improve the security of each one among our homes/workplaces/any. The general process occurs the use of the usage of . The is more correct than the Eigen Faces method [Modela Gopi-2017][4]. The author Sejal Shah et al., explains that Face Detection and Face Recognition is the biometric on which lot of work has been finished over the numerous years. 
Over the time lot of $\mathrm{s}$ are added for detection and popularity which might be taken into consideration because it is an enhanced manner for person identity and does not require Human cooperation for figuring out or verifying. Viola-Jones detection is a for detection of facial parts. It is an effective and fast way of detection than any gift methods.The system depicts the method of face detection and reputation by using PCA for Eigen faces made of snap shots within the database and the only captured or one within the database. First method is extraction of some simple parts of a face. The date may be the only stored inside the database. This is done the usage of Viola Jones . The Second technique is based totally on Principal Component Analysis technique. In this approach the records which defines more features approximately the face more is extracted. Face can be represented in-phrases of the Eigen price coordinate system [Sejal Shah-2017][5].

In the system author Harnani Hassan et al., explains about the functionality of the designed machine showed the transmission of statistics from GUI to micro-controller is effectively carried out after the photograph is recognized. The system can be progressed to end up absolutely computerized face recognition machine by adding another feature that routinely capture image with a sensor when there is human present at the doorway area. It was done by micro-controller for matching the facial details to the admin data [Harnani Hassan-2012][6].

The author bock et al., explains about the Face Recognition System for Automated Door Opening with parallel Health Status Validation Developing gadget. In this paper, a combined machine is proposed providing knee function acquisition while strolling on stairs in addition to face recognition based automatic door opening. This technology not only opens the door it will scan the knee positions body structure and scanning of the total body [BOCK-2016][7].

The author N. Paramar et al., explains that it extracts its features. It recognizes all, no matter the lighting, expression, illumination, ageing, transformations (translate, rotate and scale image) and pose, that is a hard task. This paper contains 3 sections. The first segment explains the not unusual methods like holistic matching approach, characteristic extraction technique and hybrid strategies. The second section explains programs with examples and finally third segment explains about the destiny research directions of face recognition [N. Parmar-2013][8].

The abdominal et al., authors explained that the face recognition is just satisfactorily been performed by this trend. He proposed the new coded software for this system in MATLAD which was founded to be $90 \%$ successful in this face recognition platform. The result is $90 \%$ accuracy successful. This approach can be implemented by the usage of some extra features to this machine. Light normalization and correct segmentation of face may additionally permit the threshold cost to improve. Cruising the warping space extra efficiently, e.G. using a corresponded face rotation and gesture geometric model, may accelerate the execution time.Future work might also include improvement of the Face-recognition the usage of unique characters inside the face (distance among eyes) and also examine the face in 3-D by the usage of the mixture of cameras and by using these two methods,the chance of error will decrease and the device might be greater accurate and with a completely low cost [abdominal - 2014][9].

\section{PROPOSED METHODOLOGY}

In our country the face recognition is mostly used in unlocking of the mobiles. It will scan the facial parameters and unlocks the mobile if it is matches with the admin face parameters. If the mismatch occurs it will again display messages to try again and again. With this system the robber will not be able to identify. This face recognition system is not implemented that much for home security systems that is for door opening and closing. The proposed methodology will capture the image of the person who stands at the door. The image is sent to the controller unit which checks the image with the data base. If it matches the door will be opened. Or else it would send a mail directly to the authorized person with the image of the unknown person. If it is suspicious, then an alarm could be generated.

This system works under the embedded c code that installed in the raspberry $\mathrm{Pi}$. When the image is scanned by the camera, the data will be sent to the raspberry Pi about the image. It will convert the image into the pixels. This pixels image will be seen whether it is matched with the admin face. If the admin face is matched, it will automatically open the door. If the face is mismatched, then it will generate an alarm and sends a alert mail to the admin mail. This work is proposed mainly to increase the security system in single households and elder families. The fingerprint sensor has also been used for additional security. The driver motor will increase the voltage and give voltage to the motor to open the door.

\section{Block diagram :-}

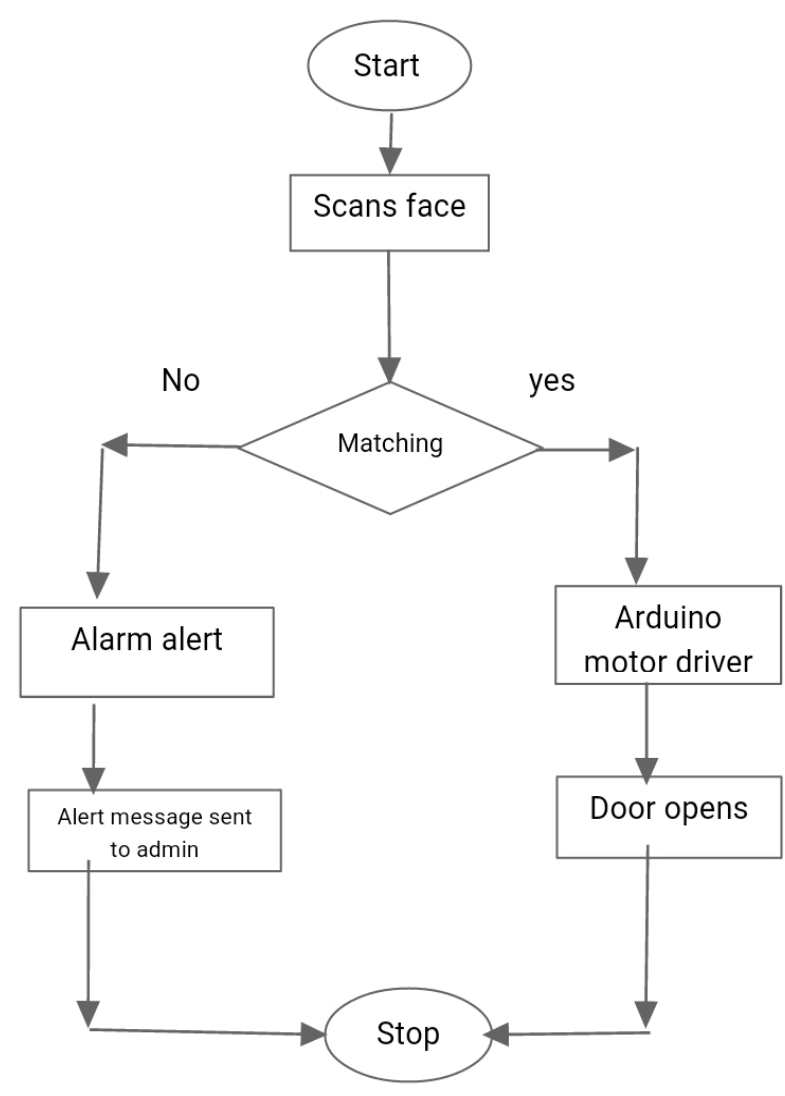

Fig.1 Block diagram of the Proposed System 
Raspberry $\mathrm{Pi}$ is a electronic device used for prototyping platform enabling users to create interactive electronic objects. They are able to read inputs - light on a sensor, a finger on a button, or a Twitter message - and turn it into an output - activating a motor, turning on an LED. Camera is an optical instrument used to capture and record images. Simple Web cameras can be arranged in circuit. Alarm is a electronic device that designed and used to alert an individual or group of individuals at a specified time. Driver motor is an interface between circuits and motor. It turns the low voltage $(5 \mathrm{v})$ to high voltage that can be used for motor. Motor is an device that it will electrical machine that converts electrical energy into mechanical energy. This motor is used to open the door. The block diagram of the project is shown in Figure.1.

\section{WORKING}

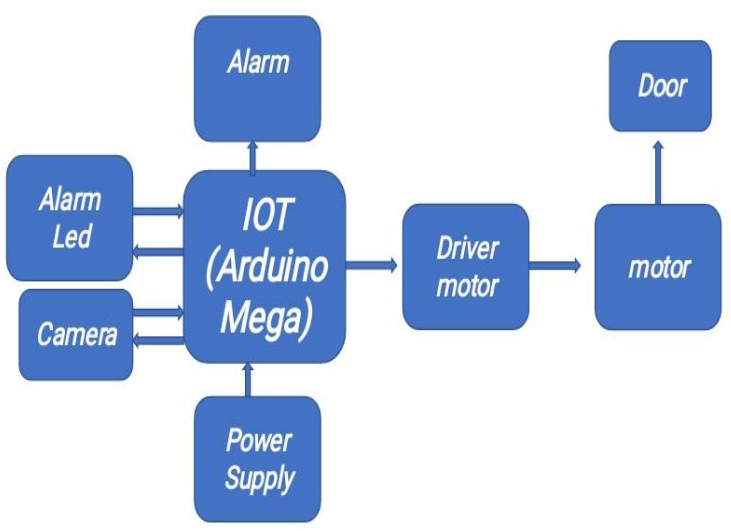

Fig.2 flowchart of the proposed system

When the power supply is on, camera captures the facial information and sends to the raspberry Pi . raspberry $\mathrm{Pi}$ will check the matching between the stored image and captured image.

When the person stands in front of the door. The camera automatically scans the face into the gray scale images. It will do the segmentation process. It divides the total image to the pixels range. Then the aurdino software will check whether the captured image is matched to admin face. The working of the total system be shown in Figure.2.

HAAR cascade algorithm is simple solution for the face recognition problem, which can be recognizing both the front face and side face. It will divide the image into cells.For each cell it contains pixels, which is converted to binary format regarding its brightness. It will split the image into many cells and it will store it as binary values or the grid $\mathrm{X}$ grid $\mathrm{y}$ values.

If the two faces matches each other then the power supply will be sent towards motor to rotate for opening the door. If the two faces are different then raspberry $\mathrm{Pi}$ will send the power supply to the alarm for warning.

\section{RESULTS AND DISCUSSION}

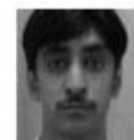

ID: 1 Aftab Ahmed

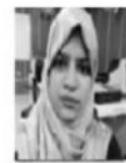

ID: 2

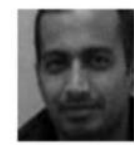

ID: 3 Jakhro

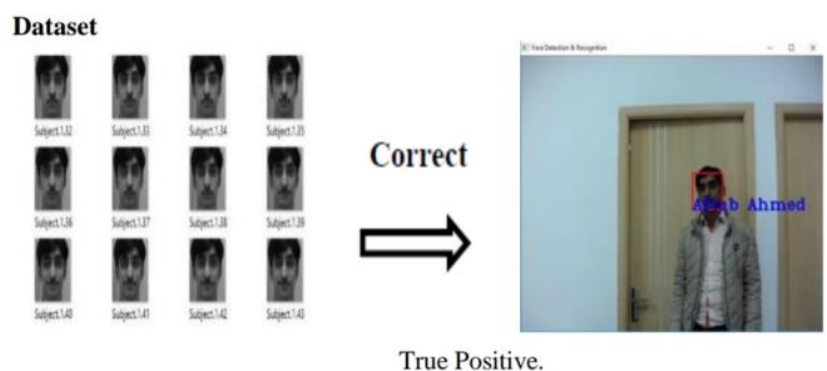

Fig.3 The sequence of performance of the test.

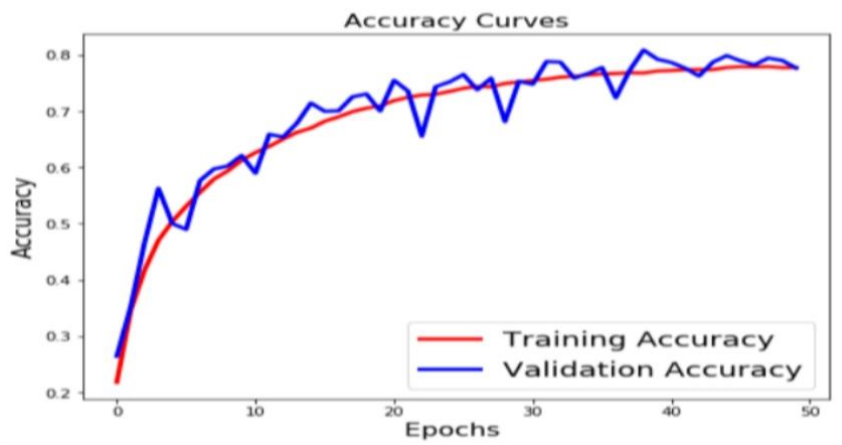

Fig.4 The performance of the test accuracy.

This system uses the home door lock access control based on face recognition method by verifying enrolled facial images. The raspberry $\mathrm{Pi}$ takes the admin facial parameters in different angles and it convert it into grayscale images for its reference. This has been developed to increase the higher security purpose in single house hold and elderly households. By adding some more features like laser security system, fingerprint sensor, keypad, it can be used as the hybrid security system uses in the banks etc. Not only for the mobiles and banks this project is modified to keep the security for every households too with the low cost. The sequence of performance of the test is shown in figure.1.

This system is always connected to the Wi-Fi using this setup the email alert system can be achieved. In this system the usage of face recognition will be along with the e-Mail alert system is designed. If any error occurs the concer person will get the email alert regarding all the information . Face recognition is one of the most important techniques for recognizing people. The performance of the test accuracy is shown in figure.2. 


\section{CONCLUSION}

In this proposed door access system by using the face recognition technology the images are stored in a data base using LPBH . This system is used door lock access for Residential purposes and Commercial Purposes. Here we have designed a highly secured door locking system by using raspberry $\mathrm{Pi}$. This paper concludes the advanced implements in the face recognition process in various places. The main purpose of the project to ensure security at the low cost.

\section{REFERENCES :-}

1. Thulluri Krishna Vamsi, Kanchana Charan Sai, Vijayalakshmi M, "Face recognition based door unlocking system using Raspberry Pi", International Journal of Advance Research, Ideas and Innovations in Technology, ISSN: 2454-132X, Impact factor: 4.295(Volume 5, Issue 2).

2. Tejas Saraf1, Ketan Shukla2, Harish Balkhande3, Ajinkya Deshmukh4, "AUTOMATED DOOR ACCESS CONTROL SYSTEM USING FACE RECOGNITION", International Research Journal of Engineering and Technology (IRJET) e-ISSN: 2395-0056, Volume: 05 Issue: 04 | Apr-2018.

3. Hteik Htar Lwin, Aung Soe Khaing, Hla Myo Tun, "Automatic Door Access System Using Face Recognition", INTERNATIONAL JOURNAL OF SCIENTIFIC \& TECHNOLOGY RESEARCH VOLUME 4, ISSUE 06, JUNE 2015.

4. Abdul Azeem, Sathuluri Mallikharjuna Rao, Kandula Rama Rao, Shaik Akbar Basha, Harsha Pedarla, Modela Gopi, "Door Unlock using Face Recognition", International Journal of Advanced Research in Electronics and Communication Engineering (IJARECE)Volume 6, Issue 4, April 2017

5. 5.Ishaan Sathe, Chiman Patel, Prasad Mahajan, Tanmay Telang, Sejal Shah,"Automatic Locking Door Using Face Recognition", International Journal of Engineering Technology Science and Research IJETSRwww.ijetsr.comISSN 2394 - 3386Volume 4, Issue 4April 2017

6. Harnani Hassan, Rosli Abu Baker, "Face recognition based on autoswitching magnetic door lock system using microcontroller" See discussions, stats, and author profiles for this publication at: https://www.researchgate.net/publication/261381471, 2012.

7. A. Ogawaa , A. Mitaa, C. Georgoulas b and T. Bockb"A Face Recognition System for Automated Door Opening with parallel Health Status Validation Using the Kinect v2 "33rd International Symposium on Automation and Robotics in Construction (ISARC 2016).

8. Divyarajsinh N. Parmar1, Brijesh B. Meta," Face Recognition Methods \& Applications", Divyarajsinh N Parmar et al ,Int.J.Computer Technology \& Applications,Vol 4 (1),84-86 IJCTA | Jan-Feb 2013 Available online@www.ijcta.com84 ISSN:2229-609.

9. Elie abdominal, "Face recognition technology" 2014See discussions, stats, and author profiles for this publication at: https://www.researchgate.net/publication 